\title{
A Simulation-Based Analysis of the Effects of Variable Prosthesis Stiffness on Interface Dynamics between the Prosthetic Socket and Residual Limb
}

Michael A. McGeehan ${ }^{1}$, PhD; Peter G. Adamczyk, PhD²; Kieran M. Nichols, MS²; Michael E. Hahn, PhD $^{1}$

Institutional Affiliations

${ }^{1}$ University of Oregon Department of Human Physiology 181 Esslinger Hall, 1525 University St.

Eugene, OR 97403

mmcgeeha@uoregon.edu, mhahn@uoregon.edu

${ }^{2}$ University of Wisconsin-Madison Department of Mechanical Engineering Room 3039, Mechanical Engineering Building

1513 University Ave.

Madison, WI 53706-1539

peter.adamczyk@wisc.edu, knichols4@wisc.edu

41

Corresponding Author Information

Michael E. Hahn, PhD

Eugene, OR 97403

(541) 346-3554

47 mhahn@uoregon.edu 
50 Introduction: Loading of a residual limb within a prosthetic socket can cause tissue

51 damage such as ulceration. Computational simulations may be useful tools for

52 estimating tissue loading within the socket, and thus provide insights into how

53 prosthesis designs affect residual limb-socket interface dynamics. The purpose of this

54 study was to model and simulate residual limb-socket interface dynamics and evaluate

55 the effects of varied prosthesis stiffness on interface dynamics during gait. Methods: A

56 spatial contact model of a residual limb-socket interface was developed and integrated

57 into a gait model with a below-knee amputation. Gait trials were simulated for four

58 subjects walking with low, medium, and high prosthesis stiffness settings. The effects of

59 prosthesis stiffness on interface kinematics, normal pressure, and shear stresses were

60 evaluated. Results: Model-predicted values were similar to those reported previously in

61 sensor-based experiments; increased stiffness resulted in greater average normal

62 pressure and shear stress $(p<0.05)$. Conclusions: These methods may be useful to aid

63 experimental studies by providing insights into the effects of varied prosthesis design

64 parameters or gait conditions on residual limb-socket interface dynamics. The current

65 results suggest that these effects may be subject-specific.

67 Key terms: Simulation, Computational model, prosthetic socket, residual limb,

68 rehabilitation 
69 Nomenclature

Acronyms widely used in text

AP

BKA

DoF

LLA

$\mathrm{ML}$

Ode23t

PD

SD

VSF
Anterior-Posterior

Below-Knee Amputation

Degrees of Freedom

Lower Limb Amputation

Medial-Lateral

Ordinary Differential Equation 23 trapezoidal solver

Proximal-Distal

Standard Deviation

Variable Stiffness Foot

\section{Abbreviations}

$\mathrm{F}$

$k$

$\mu$

$v$

$\delta$

$\dot{\delta}$
Force, $\mathrm{N}$

Linear stiffness, $\mathrm{N} / \mathrm{mm}$

Coefficient of friction

Linear velocity $\mathrm{m} / \mathrm{s}$

Penetration depth, $\mathrm{m}$

Penetration velocity, $\mathrm{m} / \mathrm{s}$

\section{Superscripts and subscripts}

$F_{f}$

$F_{n}$

$v_{p o c}$

Vthreshold

$\mu_{\text {kinetic }}$

$\mu_{\text {static }}$
Frictional force, $\mathrm{N}$

Normal force, $\mathrm{N}$

Linear velocity at point of contact, $\mathrm{m} / \mathrm{s}$

Linear velocity threshold, $\mathrm{m} / \mathrm{s}$

Coefficient of kinetic friction

Coefficient of static friction 


\subsection{Introduction}

Rehabilitation following a lower limb amputation (LLA) often includes prescription of a prosthesis designed to replace the functionality of the removed limb. For an individual with a below-knee amputation (BKA), a prosthesis system typically consists of a socket, which interfaces with the residual limb, a rigid pylon, and a foot-ankle prosthesis. Use of lower limb prostheses can improve mobility, health, and quality of life. However, abnormal loading of the soft tissues surrounding the truncated shank (e.g., asymmetric pressure distribution and elevated shear forces) can cause tissue deformation and ischemia during load bearing activities ${ }^{1}$. These conditions can lead to cell death, tissue damage, and give rise to ulceration and pain².

Dermatological issues are experienced by $75 \%$ of individuals using lower limb prostheses ${ }^{2,3}$ over their lifetime. These conditions lead to prosthesis disuse ${ }^{4}$ and can cause an individual with BKA to become wheelchair-bound. Recent estimates suggest that $11-22 \%$ of individuals abandon their prosthesis within one year of prescription ${ }^{5}$. These data are supported by a report which found that $25 \%$ of users abandoned prosthetic limbs, with $29 \%$ citing discomfort, $25 \%$ citing pain, and $12 \%$ claiming poor fit as the determining factor ${ }^{6}$. This represents a substantial reduction in quality of life and increased healthcare-related financial burden.

The socket is a crucial component of mobility and quality of life for individuals with BKA due to its role as the interface between the human, prosthesis system, and gait environment. An improved understanding of biomechanical interaction between the residual limb and prosthetic socket during gait is necessary to attenuate rates of tissue damage and prosthesis disuse. Previous experiments evaluating residual limb-socket interface dynamics have relied on sensors integrated into the prosthetic socket ${ }^{7-12}$. However, previous systems have utilized bulky sensors $^{9,10,12}$, tethered cables $^{8-11,13-16}$, or required modifications to the socket ${ }^{11,12}$, thereby compromising the integrity of the socket interface and likely altering gait mechanics of participants.

Simulations based on computational models may be useful for evaluating the relationships between anatomical morphologies, gait mechanics, design of prosthesis systems, and residual limb loading conditions. Previous model-based research has primarily employed finite element (FE) analysis techniques to derive dynamic mathematical models of the residual limb-socket interface ${ }^{1,11,17,18}$. While FE models allow for complex characterization of the biological materials and their mechanical properties, they require individual-specific imaging data as inputs, which is a cost and procedure not currently part of typical treatments. They are also computationally costly and thus may be prohibitive when integrated with complex gait models or models of other complex systems (e.g., biomechatronic rehabilitation devices). This computational cost may restrict usability in clinical or personalized medicine scenarios. Other studies have used abstract representations of the interface, such as an idealized joint parameterized with spring and damper force laws ${ }^{19}$. These methods may be appropriate for estimating generalized residual limb kinematics within the socket, but are unable to differentiate limb-socket interaction forces and torques and offer little insight regarding relative load distribution at different anatomical locations around the limb. Thus, there remains a need for a computationally economical model of the biomechanical contact forces arising from the residual limb-socket interaction during gait. 
This paper presents the design and development of a spatial contact force model motivated by the material properties of the residual limb and prosthetic socket. The contact model was integrated into a larger computational gait model to simulate kinematics and kinetics at the socket interface during gait with a semi-active variable-stiffness prosthesis. We simulated gait with three stiffness settings of the prosthesis, driven with subject-specific human experiment data, to determine how foot parameters affect limb-socket interface dynamics. It was hypothesized that the lowest limb-socket shear and pressure values would occur in the low stiffness trials. Similarly, it was hypothesized that the lowest axial rotation and vertical displacement of the residual limb inside the socket would occur in the low stiffness trials. This model could assist experimental studies by providing insight into the effects of varied prosthesis design parameters or gait conditions on residual limb-socket interface dynamics.

\subsection{Materials and Methods}

\section{$128 \quad 2.1$ Model Design}

129

A spatial contact model of the residual limb-socket interface was developed in Simscape Multibody (Mathworks Inc, Natick, MA). The geometry of the residual limb bone element was simplified as a rectangular cuboid with struts to represent the dimensions of the limb inclusive of the soft tissue (Figure 1). Within the residual limb model, soft tissue and bone element mechanics are not differentiated (i.e., the modeled dynamics are considered to be an aggregate of soft tissue and bone mechanics). The prosthetic socket was modeled as a rigid hollow square cone with an aperture of $100 \mathrm{deg}$. The residual limb interfaces with the socket at the same angle. The residual limb and socket have nine interface frames with attached cuboid structures to model interface dynamics. The shape of this model simplifies the continuous geometry of the residuum and prosthetic socket, while allowing resolution of force distribution among the different interface frames and aspects of the residuum.

The mass of the residual limb was estimated by deriving estimated density of the intact limb, modeled as a conical frustum with an assigned mass estimated per De Leva (1996) ${ }^{20}$. The derived density of the intact limb was applied to the residual limb model, which was then truncated at the respective level of amputation for each subject (Figure 1). The mass of the residuum was distributed evenly as point masses about the nine interface frames. The residuum has two contact interfaces (one proximal, one distal) on each of the four sides of the cuboid. The ninth interface is between the distal limb and base of the prosthetic socket. The distance between the distal residual limb and base of the socket was assumed to be $1.5 \mathrm{~cm}^{21}$, representative of an air gap, which is common between the socket and liner in prosthetic socket systems ${ }^{21}$. Figure 1 depicts the rotationally symmetrical model.

Contact forces at the interfaces between the limb and socket in the normal plane are implemented as modified Kelvin-Voigt material models with progressive spring and damper characteristics. Shear stresses between the socket and residual limb are considered analogous to the frictional forces arising from these interactions. In total, the residual limb has 4 degrees of freedom (DoF) with respect to the prosthetic socket, including vertical translation (i.e., pistoning) and rotations about three axes. 
A Kelvin-Voigt material model (spring and damper force law) was implemented to estimate residual limb-prosthetic socket interaction forces. The model estimates normal $\left(F_{n}\right)$ and frictional forces $\left(F_{f}\right)$ associated with the contact between a viscoelastic residual limb (spring and damper system) and rigid prosthetic socket (eq. 1). The present model does not include a socket-liner interface, but one could be implemented in the future. The spring force $(k)$ increases as a function of penetration depth $(\delta)$, whereas damping force $(b)$ increases with penetration velocity $(\dot{\delta})$. Damping force is only applied when $\dot{\delta}>0$. Frictional forces are calculated as the product of normal force and a user-defined coefficient of friction ( $\mu$ ) (eq. 2). A stick-slip friction law defines the transition between static ( $\left.\mu_{\text {static }}\right)$ and kinetic $\left(\mu_{\text {kinetic }}\right)$ coefficients of friction based on a velocity threshold $\left(v_{\text {thresh }}\right)$. Forces are applied along a common contact plane and conform to Newton's Third Law of Motion.

Values for spring stiffness in the normal plane $\left(k_{n}\right)$ were formulated according to Hooke's Law (eq. 3), as described in Zheng et al. (1999) ${ }^{22}$ and Noll et al. (2017) ${ }^{23}$. The effective tissue moduli for individuals with a below-knee LLA (Table 1) were previously described in Zheng et al. (1999) ${ }^{22}$ and Mak et al. (1994) ${ }^{24}$. In both studies, Poisson's ratio was assumed to be 0.45. The stiffness values were parameterized independently for the anterior, posterior, medial, lateral, and distal contact interfaces to best represent the varying moduli at corresponding anatomical locations. Due to a lack of information reported in previous literature, damping coefficients $(\mathrm{N} \cdot \mathrm{s} / \mathrm{mm})$ were set to half the numerical value of stiffness $(\mathrm{N} / \mathrm{mm})$ in an effort to reduce high frequency oscillations at the contact interfaces, which can lead to rapidly-evolving ordinary differential equations, and thus computational instability when simulating interface dynamics.

The static coefficient of friction ( $\mu_{\text {static }}$ ) between the limb and socket was assigned a value of 0.5 , based on an in vivo study of the interaction between silicone rubber (a commonly used material for prosthetic socket liners) and the skin of the human leg ${ }^{26}$. Coefficients of friction between 0.5 and 3.0 have been reported for various other socket liner materials ${ }^{27,28}$. The dynamic coefficient of friction ( $\mu_{\text {dynamic }}$ ) was set to $70 \%$ of the $\mu_{\text {static }}$ based on Cagle et al. $(2018)^{29}$. A velocity threshold $\left(\mu_{\mathrm{vth}}\right)$ of $0.005 \mathrm{~m} / \mathrm{s}$ defines the transition between the two values. In the future, subject-specific values for $\mu_{\text {static }}$ and $\mu_{\text {dynamic }}$ could be implemented.

The model predicts normal pressure and shear stress at the contact interfaces. Based on these forces, relative kinematics between the residual limb and prosthetic socket are simulated. Model-derived estimates may be compared to the range of experimental values reported in the literature for pressure, shear stress, and residual limb kinematics $7,8,10,14,17,19,30,31$. Previouslyreported peak values for normal pressure range from $40-160 \mathrm{kPa}^{8,15,30}$, and peak values for shear stress range from 3-50 $\mathrm{kPa}^{7,10,11,23}$. The broad range of values in the literature may be attributed to variation in sensors used, sensor placement, socket materials, individual-specific residual limb tissue properties, and experimental gait protocols. Values should vary based on phase of the gait cycle and anatomical location ${ }^{1,7,8,23}$. Nevertheless, values within these ranges may be used as target criteria. Values of 1.0 to $4.2 \mathrm{~cm}$ have been reported for relative vertical translation (i.e., residual limb pistoning) $19,31-33$. These values may vary based on residual limb shape ${ }^{34}$ and type of socket liner used ${ }^{31-33}$ and whether a vacuum or pin lock is incorporated in the prosthesis attachment. Reported values for axial internal/external rotation (rotation about the residual limb's long axis) are between 0 and \pm 20 deg during gait ${ }^{19}$. 
Manuscript Body

201

202

203

204

205

206

207

208

209

210

211

212

213

214

215

216

217

218

219

220

221

222

223

224

225

226

227

228

229

230

231

232

233

234

235

236

237

238

239

240

241

242

243

244

\subsection{Gait Simulations with Experimental Data}

The spatial contact model of the residual limb-socket interface was integrated into a gait model with a BKA and a semi-active variable stiffness foot (VSF) prosthesis. This model was previously described in McGeehan et al. (2021a and 2021b) ${ }^{35,36}$. Briefly, gait simulations were driven by experimental data from four individuals (Table 2) walking with the VSF $^{37}$ configured to "high", "medium", and "low" stiffness settings, corresponding to forefoot stiffness values of 10, 19 , and $32 \mathrm{~N} / \mathrm{mm}$. Forward kinematics simulations were computed for three trials per setting, as in McGeehan et al. $(2021 b)^{36}$. Subject 2 did not complete one medium stiffness trial, and Subject 4 did not complete one high stiffness trial. In total, 34 simulations were computed. All simulations were computed in Simscape Multibody using the ode23t solver profile with variable step sizes for numerical integration.

The experimental motion capture data used to drive the model were insufficient to estimate kinematics of the residual limb with respect to the prosthetic socket. As such, data from the literature were used to constrain motion of the residual limb via a bearing joint. A progressive spring and damper force law was used to constrain motion. Limits of $+0.5 \mathrm{~cm}$ (upward displacement) and $-3.5 \mathrm{~cm}$ (downward displacement) were imposed for residual limb vertical translation ${ }^{19,31-33,38}$. Constraints of $\pm 10 \mathrm{deg}, \pm 5 \mathrm{deg}$, and $\pm 5 \mathrm{deg}$ were imposed for axial rotation, anterior-posterior $(\mathrm{AP})$, and medial-lateral $(\mathrm{ML})$ rotations ${ }^{19,39}$. These constraints were necessary to account for the differences in contact surface areas between the discretized contact model and the continuous geometry of a biological residual limb and its mechanical interface with a prosthetic socket.

\subsection{Data Processing and Statistical Methodology}

Kinematic and kinetic signals related to the simulated limb-socket interface were lowpass filtered via zero-lag a $4^{\text {th }}$ order Butterworth $\left(f_{c}: 6 \mathrm{~Hz}\right)$. Data were indexed from heel strike to toe-off and resampled to 101 data points via cubic spline interpolation. These methods allow for comparison of stance phases of different lengths. Model-derived values were compared to those previously reported in the literature and the effects of stiffness setting on limb-socket dynamics were evaluated using linear mixed effects (LME) regression analysis. It was hypothesized that these effects would be subject-dependent, and as such, an exemplary case study for Subject 1 is presented along with group level data.

We computed discrete outcome metrics from the simulations. Interfacial normal forces, frictional forces, pistoning displacement, and axial rotations were derived from the spatial contact model for the duration of stance phase. From the simulated forces, normalized pressure values were calculated for each of the nine interface frames based on the surface area of the interface and the body weight (BW) of the participant $(\mathrm{kPa} / \mathrm{BW})$. Pressure distributions between opposing frames ( $A P, M L$, and proximal-distal (PD)) were calculated as the percent contribution of each contact interface to the sum of the pressure of both opposing interfaces. Peak values for each interface and peak average values (i.e., peak of the average value for the nine interfaces) were reported and used as dependent variable for subsequent analyses.

The relationships between the aforementioned outcome variables and prosthetic foot stiffness condition were addressed using LME regression. For each analysis, the peak of the simulated outcome variable was the dependent variable, stiffness setting was the independent 
variable, and subject was a random effect. We computed the least squares coefficients to the linear mixed effects model (metric $=\beta_{0}{ }^{*}$ stiffness $+O_{i}$ ), along with confidence intervals and $\mathrm{p}$-values for each coefficient. The random effect appears as a unique intercept $\left(O_{i}\right)$ for each subject. The overall effect of stiffness on each outcome measure (coefficient $\beta_{0}$ ) was evaluated $(\alpha=0.05)$. We report the slope, mean intercept, adjusted $\mathrm{R}^{2}$, and $\mathrm{p}$-value for each model. The slope $\left(\beta_{0}\right)$ represents the sensitivity of the outcome to changes in stiffness, whereas the mean intercept (mean of all $O_{i}$ ) allows for characterization of the group average values. The adjusted $\mathrm{R}^{2}$ value represents the strength of the independent variable to explain variations in the dependent variable, excluding the effect of individual intercepts. P-value quantifies the statistical confidence that the slope (sensitivity) is different from zero. These methods were adapted from previous work evaluating the effects of prosthesis design on gait outcomes ${ }^{40-42}$. Analyses were performed using MATLAB 2020b (Mathworks Inc., Natick, MA).

\subsection{Results}

\subsection{Model Performance (Group Data)}

Contact model-derived values for normal pressure and shear stress were dependent upon anatomical location (Table 3) and progression of stance phase (Figures 2 and 3). Peak average normal pressure across stance phase was $70.4 \pm 4.28,75.9 \pm 4.44$, and $85.0 \pm 13.0 \mathrm{kPa}$ for the low, medium, and high stiffness conditions, whereas peak average shear stress values were $25.0 \pm 1.52,26.6 \pm 1.55$, and $29.9 \pm 4.61 \mathrm{kPa}$ for the same conditions (Figure 2, Table 5). Increased prosthesis stiffness was associated with increased peak average normal pressure and shear stress $(p<0.05)$ (Figure 4, Table 3); specific increases were observed at the anterior proximal and anterior distal interfaces $(p<0.05)$. Stiffness did not significantly affect normal pressure or shear stress at the other interface frames, nor did it affect residual limb pistoning or axial rotation $(p>0.05)$, though effects were subject-dependent (Table 3 ).

Spatiotemporal patterns for pressure and shear stress distribution were variable between participants, but show similar variability across stiffness conditions (Figure 3, Tables 4 and 5). On average, participants displayed predominantly anterior pressure and shear distributions early in stance phase, but trended toward a more even distribution later in stance phase. Pressure trended slightly toward the lateral and proximal aspects of the residual limb thoughout stance phase. High variability was observed among participants for the AP and ML distributions throughout stance phase.

\subsection{Case Study (Subject 1)}

Mean data for Subject 1 demonstrated less variability compared to the group data (Figures 5 and 6). Average pressure and shear stress values peaked at approximately $20 \%$ stance phase, whereas residual limb pistoning peaked and plateaued near $50 \%$ stance phase. Maximal pistoning displacement was approximately $1.5 \mathrm{~cm}$ for all conditions. A slightly increased rate of pistoning appeared between 15-40\% stance phase for the high stiffness compared to the low and medium stiffness conditions (Figures 5 and 6). The subject's residuum was predominately externally rotated with respect to the prosthetic socket throughout stance phase with maximal external rotation occurring near $50 \%$ stance phase. The low stiffness 
condition resulted in the least external rotation, though high variability was observed late in stance phase for all conditions.

The effects of variable prosthesis stiffness on pressure and shear stress distribution appeared primarily during $50-100 \%$ of stance phase (Figure 6). However, divergent patterns in the mean data were accompanied by greater variability during this time. From $0-50 \%$ stance phase, pressure and shear stress were weighted more heavily toward the anterior aspect of the residual limb for all stiffness conditions. For the low and high stiffness conditions, mean pressure and shear stress trended toward a relatively even AP distribution late in stance phase, whereas the medium stiffness condition resulted in a relatively posterior distribution. Pressure and shear stress distribution outcomes were similar across stiffness conditions for the ML and proximal-distal aspects of the residuum.

\subsection{Discussion}

\subsection{Model Performance (Group Data)}

The objective of this study was to develop a spatial contact model of the residual limbprosthetic socket interface and evaluate its ability to estimate limb-socket interface dynamics. A secondary objective of this study was to use this model to examine the relationships between prosthetic foot stiffness and limb-socket dynamics. The hypothesis that limb-socket normal pressure and shear stresses would be lowest in the low stiffness condition was supported for the average normal force and shear stress metrics. Specific effects were observed at the anterior proximal and anterior distal interfaces. Stiffness did not significantly affect normal pressure or shear stress at the other interface frames, nor did it affect residual limb kinematics.

Average normal pressure and shear stress increased with prosthesis stiffness during stance phase. This pattern may indicate the need for greater force transfer from the residuum to the prosthetic socket to load a stiffer prosthetic foot. This is supported by the specific increases observed at the anterior interfaces. Participants may have adopted a strategy whereby they increased the anterior forces in the socket to deform the energy storage and return structures of the VSF under stiffer configurations. Increased prosthesis stiffness has been shown to offer potential biomechanical benefits such as increased mechanical efficiency ${ }^{43}$ and increased propulsion ${ }^{43}$. However, these data suggest that prosthesis users may in part achieve this through increased loading of the limb-socket interface, which may increase risk of tissue damage via friction or pressure ulcers.

The pressure distribution profiles derived from this model were weighted toward the anterior and lateral aspects of the residual limb. These patterns may be due to gait kinematics of the participants. Future clinical gait evaluations should seek to verify these patterns.

Model-derived values for normal pressure depict spatiotemporal patterns similar to those of a ground reaction force (GRF) curve during stance phase. The pressure and shear waveforms presented by Sanders et al. (1992) $)^{45}$ and Laszczak et al. (2016) ${ }^{46}$ are similar to those of the present study early in stance phase, but exhibit a brief plateau during mid-stance before values decrease. Comparatively, the present study shows similar loading rates, but a gradual decline in pressure and stress rather than a mid-stance phase plateau (i.e., the waveforms are skewed toward early stance phase) (Figure 2). The lack of a plateau in pressure and shear data in the present results may reflect the different prostheses used for experimental gait trials. Mathematically, it could also be due to inadequate constraining of residual limb motion. Using 
experimental kinematic data to constrain residual limb motion may help refine the trajectory of the modeled response, or a velocity constraint could be implemented into the present model design. Peak values for normal pressure were similar to those reported in previous sensorbased experiments $\mathrm{s}^{8,10,30,44}$ and finite element modeling analyses ${ }^{1,17,18}$, which ranged from 40$160 \mathrm{kPa}$. Values for shear stress were within the 3-50 kPa range reported previously $7,10,11,23$.

The accuracy of the model to predict pressure and shear stress values specific to different anatomical locations is difficult to discern based on previous experiments. Sensorbased experiments have typically sampled from small localized areas on the limb or present only resultant data. Nevertheless, broad comparisons can be made with data from Sanders et al. (1992 and 2000) 30,45 and Courtney et al. (2016) ${ }^{8}$. Results of this study showed peak mean pressure and stress values on the medial side of 60 and $21 \mathrm{kPa}$ (values are the mean of the proximal and distal interfaces under all stiffness conditions). Courtney et al. (2016) reported peak medial pressure of approximately 65-70 kPa, whereas Sanders et al. (2000) present values ranging $40-85 \mathrm{kPa}$ for pressure and $7-12 \mathrm{kPa}$ for shear stress. On the lateral side, results of this study showed peak mean pressure and shear stress values of 117 and $41 \mathrm{kPa}$. Comparatively, Sanders et al. (2000) present values of 60-140 kPa for pressure and 18-23 kPa for shear stress. Posteriorly, the pressure and shear values of 41 and $13 \mathrm{kPa}$ were lower compared to those presented by Sanders et al. (85-100 and 17-22 kPa). This discrepancy may be due to the increased stiffness of the tissue on the posterior residual limb associated with muscle contraction during gait, which is unaccounted for in this model. Muscular contraction has been shown to increase tissue modulus, for example by $45 \mathrm{kPa}^{22}$ in the muscles of the forearm. Muscular contraction would likely have minimal effects on the frictional characteristics of the tissue. In the future, a progressive model of tissue moduli could be implemented into a limbsocket contact model. The model's predicted anterior pressure and shear stress were 235 and $83 \mathrm{kPa}$, which were similar to values of 245 and 105 derived from FEA of socket interface dynamics at the patellar tendon ${ }^{18}$. There is a paucity of data from sensor-based experiments related to pressure or shear dynamics along the anterior tibia.

The model predicted peak residual limb displacements between 1.2 and $2.1 \mathrm{~cm}$ with respect to the socket. These values are within the range of $1.1-3.6 \mathrm{~cm}$ (mean: $2.3 \pm 1.0 \mathrm{~cm}$ ) previously reported in the literature ${ }^{19,32-34,47-49}$. It should be noted that the prosthetic socket components (e.g., liner and socket materials) and gait tasks varied among these studies. Data from the present study, among others, support the idea that the amount of residual limb pistoning may be affected by liner and socket type ${ }^{30,50}$, residual limb shape ${ }^{34}$, and gait conditions ${ }^{30,33}$. Data regarding the prosthetic socket componentry used by participants in this study were not available.

\subsection{Case Study (Subject 1)}

Across the stiffness conditions, data from Subject 1 showed similar spatiotemporal patterns between $0-50 \%$ stance phase (Figure 5), which encompasses the progression from heel strike to foot flat ${ }^{51}$. Divergent responses were observed across the stiffness conditions in the latter half of stance phase for residual limb axial angle and AP pressure and shear stress distribution. Increased variability was also observed for all conditions during this time. The latter half of stance phase is characterized by the progression from foot flat to toe off and involves an anterior shift in the center of pressure (COP) ${ }^{51}$. Since the stiffness behavior of the 
VSF's heel is unchanged across the conditions, it is logical that the effects of variable forefoot stiffness would be primarily observed in the latter half of stance phase.

The subject presented no discernable effect of variable stiffness on the peak average

\subsection{Limitations and Future Directions}

The present model was parameterized using previously reported residual limb tissue mechanical properties and limb-socket kinematics for individuals with BKA. While these methods resulted in pressure and shear stress values within the range reported in the literature, variability in the aforementioned parameters is well documented between individual subjects. Future work should strive toward individualized models by characterizing the unique tissue mechanical properties of subjects. This could be accomplished by measuring tissue stiffness at corresponding sites between the residuum and model and using those measurements to parameterize the model. Similarly, adjusting parameters based on the socket componentry used by subjects would improve the accuracy of the model. For example, coefficients of friction between 0.5-3.0 have been reported for the interaction between human skin and various socket liner materials ${ }^{27,28}$. Variation within this range would have a substantial impact on model-derived shear stresses estimates. Further, this model does not account for frictional forces at the liner-socket interface. If frictional coefficients are lower for this interface compared to the skin-liner interface, inaccuracies in modeled shear stresses would arise.

Future work should also seek to quantify kinematics between the residual limb and prosthetic socket through optical motion capture or instrumenting participants with potentiometers. Using these data to constrain residual limb motion during simulations would improve accuracy on an individualized basis. These data could be used to refine the ability of the current model to predict limb-socket kinematics.

The present model assumes oversimplified geometries of the residuum and prosthetic socket. Developing more complex interface geometry could improve model fidelity. For example, using a pentagonal prism shape to model the residual limb geometry would allow for differentiation of the varying moduli of the anterior, anterior lateral, anterior medial, posterior lateral, and posterior medial aspects of the residual limb and would only add two interface frames compared to the present model.

This study modeled the residual limb as composite of both the bone and soft tissue elements. However, data from $x$-ray ${ }^{54}$ and biplane fluoroscopy ${ }^{55}$ studies suggest that residual limb kinematics can be differentiated into the motion of the bone and soft tissue elements. As 
421 such, it may be important to distinguish these elements and model the interface between them

422 in future studies. Doing so could lead to improvements when simulating limb-socket dynamics.

423

424

425

426

427

428

429

430

431

432

433

434

435

436

437

438

439

\subsection{Conclusions}

Findings from these simulations support the use of reduced order modeling techniques to estimate residual limb-prosthetic socket interfacial pressure and shear stress, as well as residual limb kinematics in a computationally economical manner. Residual limb-prosthetic socket interface dynamics derived from this model were within the range of values reported by previous sensor-based gait experiments. These methods may be useful to aid experimental studies by providing insights into the effects of varied prosthesis design parameters or gait conditions on residual limb-socket interface dynamics.

Simulated data showed increased peak average values for normal pressure and shear stress with a stiffer prosthesis; specific effects were observed on the anterior aspect of the residual limb-socket interface. Data from a case study show promise for evaluating individualized effects of prosthesis stiffness on limb-socket dynamics. Future work could add complexity to the modeled interface geometry in order to better match the shape and variation in tissue material properties of the residual limb. Additionally, the model's accuracy could be improved by applying subject-specific data for residual limb tissue properties and prosthetic socket componentry when parameterizing the contact interfaces. 


\section{References}

1 Portnoy S, Siev-Ner I, Shabshin N, et al. Patient-specific analyses of deep tissue loads post transtibial amputation in residual limbs of multiple prosthetic users. J Biomech 2009; 42:2686-2693. Doi: 10.1016/j.jbiomech.2009.08.019.

2 Highsmith JT, Highsmith MJ. Common skin pathology in LE prosthesis users. J Am Acad Physician Assist 2007; 20(11):33-36. Doi: 10.1097/01720610-200711000-00018.

3 Highsmith MJ, Kahle JT, Klenow TD, et al. Interventions to manage residual limb ulceration due to prosthetic use in individuals with lower extremity amputaiton: A systematic review of the literature. Technol Innov 2016; 18(3):115-123. Doi: 10.21300/18.2-3.2016.115.

4 Meulenbelt HEJ, Dijkstra PU, Jonkman MF, et al. Skin problems in lower limb amputees: A systematic review. Disabil Rehabil 2006:603-608. Doi: 10.1080/09638280500277032.

5 Balk EM, Gazula A, Markozannes G, et al. Lower Limb Prostheses: Measurement Instruments, Comparison of Component Effects by Subgroups, and Long-Term Outcomes. 2018.

$6 \quad$ National Health Services Audit Commission Briefing: Assisting Independence. 2002.

7 Sanders JE, Daly CH. Measurement of Stresses in Three Orthogonal Directions at the Residual Limb-Prosthetic Socket Interface. IEEE Trans Rehabil Eng 1993; 1(2):79-85. Doi: 10.1109/86.242421.

8 Courtney A, Orendurff MS, Buis A. Effect of alignment perturbations in a trans-tibial prosthesis user: A pilot study. J Rehabil Med 2016; 48(4):396-401. Doi: 10.2340/16501977-2075.

$9 \quad$ Laszczak P, Jiang L, Bader DL, et al. Development and validation of a 3D-printed interfacial stress sensor for prosthetic applications. Med Eng Phys 2015; 37(1):132-137. Doi: 10.1016/j.medengphy.2014.10.002.

10 Laszczak P, Mcgrath M, Tang J, et al. A pressure and shear sensor system for stress measurement at lower limb residuum/socket interface. Med Eng Phys 2016; 38:695-700. Doi: 10.1016/j.medengphy.2016.04.007.

11 Schiff A, Havey R, Carandang G, et al. Quantification of Shear Stresses Within a Transtibial Prosthetic Socket. Foot Ankle Int 2014; 35(8):779-782. Doi: 10.1177/1071100714535201.

12 Al-Fakih EA, Osman NAA, Eshraghi A, et al. The capability of fiber Bragg grating sensors to measure amputees' trans-tibial stump/socket interface pressures. Sensors (Switzerland) 2013; 13(8):10348-10357. Doi: 10.3390/s130810348.

13 Safari MR, Tafti N, Aminian G. Socket Interface Pressure and Amputee Reported Outcomes for Comfortable and Uncomfortable Conditions of Patellar Tendon Bearing Socket: A Pilot Study. Assist Technol 2015; 27(1):24-31. Doi: 10.1080/10400435.2014.949016.

14 Gholizadeh H, Azuan N, Osman A, et al. Clinical implication of interface pressure for a new prosthetic suspension system. Biomed Eng Online 2014; 13(89). Doi: 10.1186/1475925X-13-89.

15 Ali S, Abu Osman NA, Eshraghi A, et al. Interface pressure in transtibial socket during ascent and descent on stairs and its effect on patient satisfaction. Clin Biomech 2013; 28(9-10):994-999. Doi: 10.1016/j.clinbiomech.2013.09.004.

16 Boutwell E, Stine R, Hansen A, et al. Effect of prosthetic gel liner thickness on gait 
biomechanics and pressure distribution within the transtibial socket. J Rehabil Res Dev 2012; 49(2):227-240. Doi: 10.1682/JRRD.2010.06.0121.

17 Jia X, Zhang M, Lee WCC. Load transfer mechanics between trans-tibial prosthetic socket and residual limb - Dynamic effects. J Biomech 2004; 37:1371-1377. Doi: 10.1016/j.jbiomech.2003.12.024.

18 Lee WCC, Zhang M, Jia X, et al. Finite element modeling of the contact interface between trans-tibial residual limb and prosthetic socket. Med Eng Phys 2004; 26:655-662. Doi: 10.1016/j.medengphy.2004.04.010.

19 LaPrè AK, Price MA, Wedge RD, et al. Approach for gait analysis in persons with limb loss including residuum and prosthesis socket dynamics. Int $j$ Numer Method Biomed Eng 2018; 34(4):e2936. Doi: 10.1002/cnm.2936.

20 De Leva P. Adjustments to zatsiorsky-seluyanov's segment inertia parameters. J Biomech 1996; 29(9):1223-1230. Doi: 10.1016/0021-9290(95)00178-6.

21 Henrikson KM, Weathersby EJ, Larsen BG, et al. An inductive sensing system to measure in-socket residual limb displacements for people using lower-limb prostheses. Sensors 2018; 18(11). Doi: 10.3390/s18113840.

22 Zheng Y, Mak AFT, Lue B. Objective assessment of limb tissue elasticity: Development of a manual indentation procedure. J Rehabil Res Dev 1999; 36(2):71-85.

23 Noll V, Eschner N, Schumacher C, et al. A physically-motivated model describing the dynamic interactions between residual limb and socket in lower limb prostheses. Curr Dir Biomed Eng 2017; 3(1):15-18. Doi: 10.1515/cdbme-2017-0004.

24 Mak AFT, George ;, Liu HW, et al. Biomechanical assessment of below-knee residual limb tissue. J Rehabil Res Dev 1994; 31(3):188-198.

25 Shan X, Otsuka S, Yakura T, et al. Morphological and mechanical properties of the human triceps surae aponeuroses taken from elderly cadavers: Implications for muscle-tendon interactions. PLoS One 2019; 14(2). Doi: 10.1371/journal.pone.0211485.

26 Zhang M, Mak AFT. Zn vivo friction properties of human skin. vol. 23. 1999.

27 Sanders JE, Greve JM, Mitchell SB, et al. Material properties of commonly-used interface materials and their static coefficients of friction with skin and socks. J Rehabil Res Dev 1998; 35(2):161-176.

28 Cagle JC, Hafner BJ, Taflin N, et al. Characterization of prosthetic liner products for people with transtibial amputation. J Prosthetics Orthot 2018; 30(4):187-199. Doi: 10.1097/JPO.0000000000000205.

29 Cagle JC, Hafner BJ, Taflin N, et al. Characterization of prosthetic liner products for people with transtibial amputation. J Prosthetics Orthot 2018; 30(4):187-199. Doi: 10.1097/JPO.0000000000000205.

30 Sanders JE, Zachariah SG, Baker AB, et al. Effects of changes in cadence, prosthetic componentry, and time on interface pressures and shear stresses of three trans-tibial amputees. Clin Biomech 2000; 15:684-694.

31 Eshraghi A, Osman NAA, Gholizadeh $\mathrm{H}$, et al. Pistoning assessment in lower limb prosthetic sockets. Prosthet Orthot Int 2012; 36(1):15-24. Doi: 10.1177/0309364611431625.

32 Sanders JE, Karchin A, Fergason JR, et al. A noncontact sensor for measurement of distal residual-limb position during walking. J Rehabil Res Dev 2006; 43(4):509-516. Doi: 
10.1682/JRRD.2004.11.0143.

33 Gholizadeh $\mathrm{H}$, Osman NAA, Lúvíksdóttir ÁG, et al. A new approach for the pistoning measurement in transtibial prosthesis. Prosthet Orthot Int 2011; 35(4):360-364. Doi: 10.1177/0309364611423130.

34 Wirta RW, Golbranson FL, Mason R, et al. Analysis of below-knee suspension systems : Effect on gait. J Rehabil Res Dev 1990; 27(4):385-396. Doi: 10.1682/JRRD.1990.10.0385.

35 McGeehan MA, Adamczyk PG, Nichols KM, et al. A Reduced-Order Computational Model of a Semi-Active Variable-Stiffness Foot Prosthesis. J Biomech Eng 2021; 143(7). Doi: 10.1115/1.4050456.

36 McGeehan M, Adamczyk P, Nichols K, et al. A Computational Gait Model with a BelowKnee Amputation and a Semi-Active Variable-Stiffness Foot Prosthesis. J Biomech Eng 2021. Doi: 10.1115/1.4052108.

37 Glanzer EM, Adamczyk PG. Design and Validation of a Semi-Active Variable Stiffness Foot Prosthesis. IEEE Trans Neural Syst Rehabil Eng 2018; 26(12):2351-2359. Doi: 10.1109/TNSRE.2018.2877962.

38 Darter BJ, Sinitski K, Wilken JM. Axial bone-socket displacement for persons with a traumatic transtibial amputation: The effect of elevated vacuum suspension at progressive body-weight loads. Prosthet Orthot Int 2016; 40(5):552-557. Doi: 10.1177/0309364615605372.

39 Laprè AK, Umberger BR, Sup F. Simulation of a powered ankle prosthesis with dynamic joint alignment. 2014 36th Annu. Int. Conf. IEEE Eng. Med. Biol. Soc. EMBC 2014. 2014.

40 Morgenroth DC, Segal AD, Zelik KE, et al. The effect of prosthetic foot push-off on mechanical loading associated with knee osteoarthritis in lower extremity amputees. Gait Posture 2011; 34(4):502-507. Doi: 10.1016/J.GAITPOST.2011.07.001.

41 Adamczyk PG, Roland M, Hahn ME. Sensitivity of Biomechanical Outcomes to Independent Variations ofHindfoot and Forefoot Stiffness in Foot Prostheses. Hum Mov Sci 2017; 54:154. Doi: 10.1016/J.HUMOV.2017.04.005.

42 Segal AD, Zelik KE, Klute GK, et al. The effects of a controlled energy storage and return prototype prosthetic foot on transtibial amputee ambulation. Hum Mov Sci 2012; 31(4):918. Doi: 10.1016/J.HUMOV.2011.08.005.

43 Fey NP, Klute GK, Neptune RR. Altering prosthetic foot stiffness influences foot and muscle function during below-knee amputee walking: A modeling and simulation analysis. J Biomech 2013; 46:637-644. Doi: 10.1016/j.jbiomech.2012.11.051.

44 Sanders JE, Daly CH, Burgess EM. Interface shear stresses during ambulation with a below-knee prosthetic limb. J Rehabil Res Dev 1992; 29(4):1-8. Doi: 10.1682/jrrd.1992.10.0001.

45 Sanders JE, Daly CH, Burgess EM. Interface shear stresses during ambulation with a below-knee prosthetic limb. J Rehabil Res Dev 1992; 29(4):1-8. Doi: 10.1682/jrrd.1992.10.0001.

46 Laszczak P, McGrath M, Tang J, et al. A pressure and shear sensor system for stress measurement at lower limb residuum/socket interface. Med Eng Phys 2016; 38(7):695700. Doi: 10.1016/j.medengphy.2016.04.007.

47 Grevsten S, Erikson U. A roentgenological study of the stump-socket contact and skeletal displacement in the PTB-suction prosthesis. Ups J Med Sci 1975; 80(1):49-57. Doi: 
10.3109/03009737509178991.

48 Narita H, Yokogushi K, Shii S, et al. Suspension effect and dynamic evaluation of the total surface bearing (TSB) trans-tibial prosthesis: A comparison with the patellar tendon bearing (PTB) trans-tibial prosthesis. Prosthet Orthot Int 1997; 21(3):175-178. Doi: 10.3109/03093649709164551.

49 Söderberg B, Ryd L, Persson BM. Roentgen Stereophotogrammetric Analysis of Motion between the Bone and the Socket in a Transtibial Amputation Prosthesis: A Case Study. J Prosthetics Orthot 2003; 15(3):95-99. Doi: 10.1097/00008526-200307000-00008.

50 Yiğiter K, Şener G, Bayar K. Comparison of the effects of patellar tendon bearing and total surface bearing sockets on prosthetic fitting and rehabilitation. Prosthet Orthot Int 2002; 26(3):206-212. Doi: 10.1080/03093640208726649.

51 Winter DA. The Biomechanics and Motor Control of Human Gait. 1991.

52 Gailey R, Allen K, Castles J, et al. Review of secondary physical conditions associated with lower-limb amputation and long-term prosthesis use. JRRD 2008; 45(1):15-30. Doi: 10.1682/JRRD.2006.11.0147.

53 Weidow J, Tranberg R, Saari T, et al. Hip and Knee Joint Rotations Differ between Patients with Medial and Lateral Knee Osteoarthritis: Gait Analysis of 30 Patients and 15 Controls. J Orthop Res 2006; 24:1890-1899. Doi: 10.1002/jor.20194.

54 Lilja M, Johansson T, Öberg T. Movement of the tibial end in a PTB prosthesis socket: A sagittal X-ray study of the PTB prosthesis. Prosthet Orthot Int 1993; 17(1):21-26. Doi: 10.3109/03093649309164351.

55 Bocobo C, Castellote J, MacKinnon D, et al. Videofluoroscopic evaluation of prosthetic fit and residual limbs following transtibial amputation. J Rehabil Res Dev 1998; 35(1):6-13. 
Figures and Tables

596

597

598

599

600

601

602

Table 2: Participant characteristics. including the nine interface frames. residual limb.

Figure 1: Depiction of the rotationally symmetrical residual limb and socket geometries,

Table 1: Summary of Young's Modulus values for various anatomical locations on the

\begin{tabular}{|c|c|c|}
\hline Anatomical location & $\begin{array}{l}\text { Effective modulus } \\
(\mathrm{kPa})\end{array}$ & $\begin{array}{l}\text { Corresponding } \\
\text { interface(s) } \\
\text { on the model }\end{array}$ \\
\hline Tibial tuberosity ${ }^{22}$ & 105 & Anterior \\
\hline Posterior tibia 25 & 30 & Posterior \\
\hline Distal tibia 22 & 60 & Distal \\
\hline Medial proximal tibia ${ }^{24}$ & 56 & Medial \\
\hline Lateral proximal tibia 22 & 78 & Lateral \\
\hline
\end{tabular}

Residual Limb Bone Element

Prosthetic Socket

Socket Contact Surface

Residual Limb Contact Surface

\begin{tabular}{lllllll}
\hline Subject & Sex & Age $(\mathbf{y})$ & $\begin{array}{l}\text { Height } \\
(\mathbf{c m})\end{array}$ & Mass $(\mathbf{k g})$ & $\begin{array}{l}\text { Amputation } \\
\text { side }\end{array}$ & $\begin{array}{l}\text { Years post- } \\
\text { amputation }\end{array}$ \\
\hline 1 & Male & 34 & 181 & 77.3 & Right & 15 \\
2 & Male & 51 & 175 & 111 & Right & 8 \\
3 & Male & 70 & 180 & 83.8 & Left & 14 \\
4 & Female & 61 & 163 & 63.8 & Right & 8 \\
Mean \pm SD & - & $54 \pm 15$ & $175 \pm 19.9$ & $84.0 \pm 19.9$ & - & $11 \pm 3.8$ \\
\hline
\end{tabular}



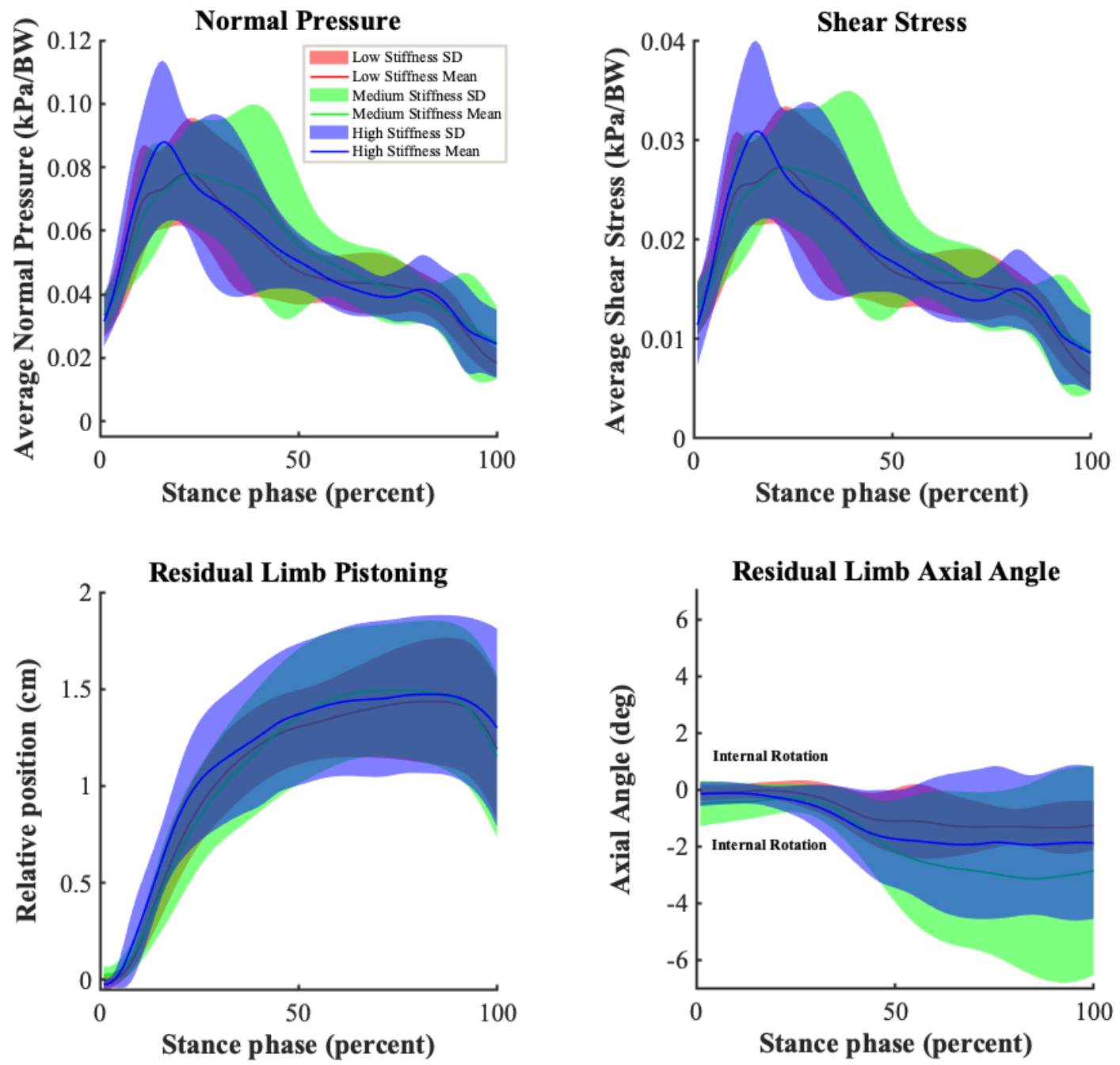

Figure 2: Group mean data for normal pressure, shear stress, residual limb pistoning, and residual limb internal/external rotation across stance phase for the low, medium, and high

607 stiffness conditions. Kinetic data are normalized to subject body weight. 
Figures and Tables
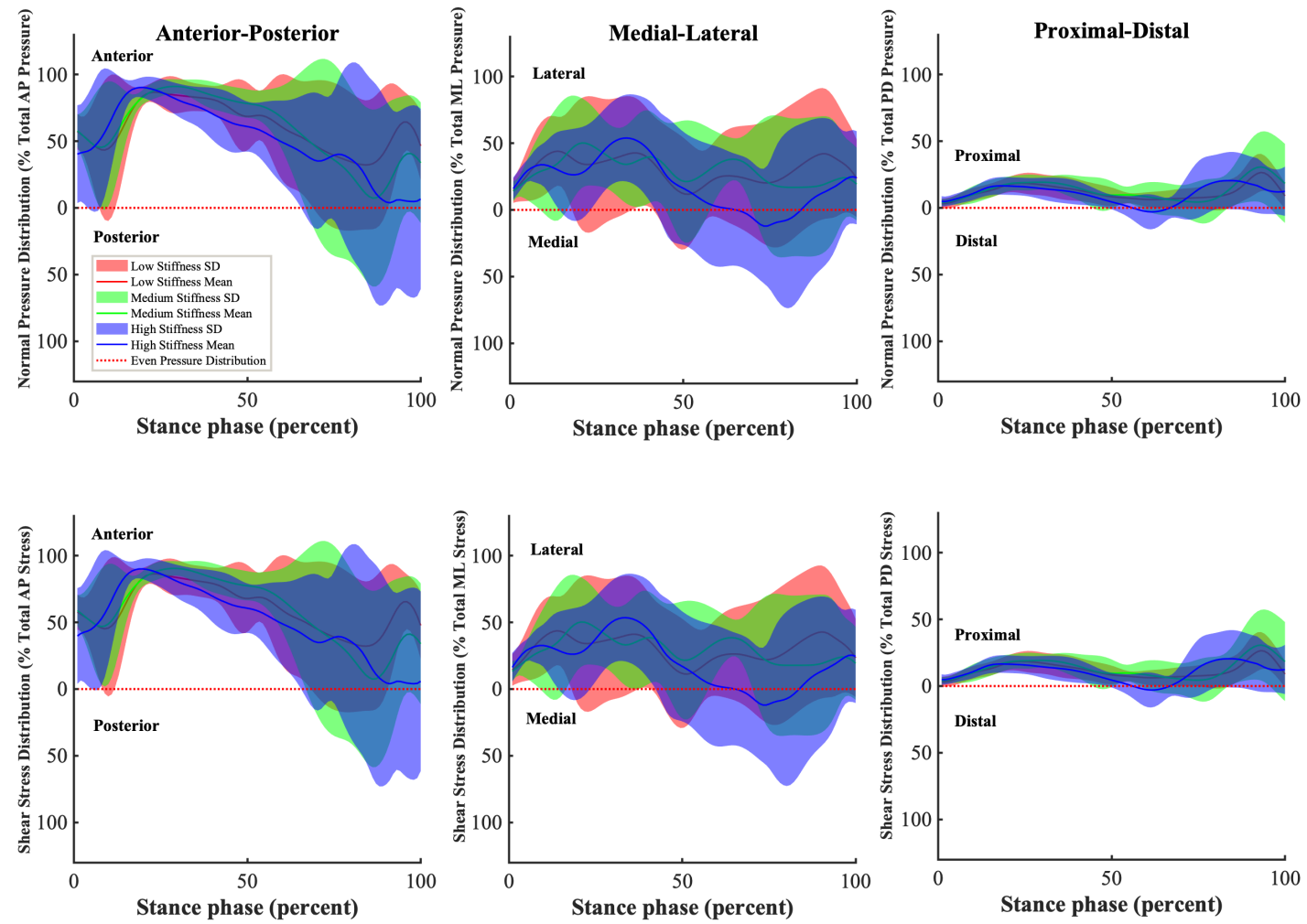

Figure 3: Group mean data for normal pressure (top) and shear stress (bottom) distributions in the anterior-posterior, medial-lateral, and proximal-distal directions. Pressure and shear stress

613 distributions are the percent contribution of opposing interface frames ( $A P, M L$, and PD) to the sum of the pressure or shear stress of the opposing the interfaces. 

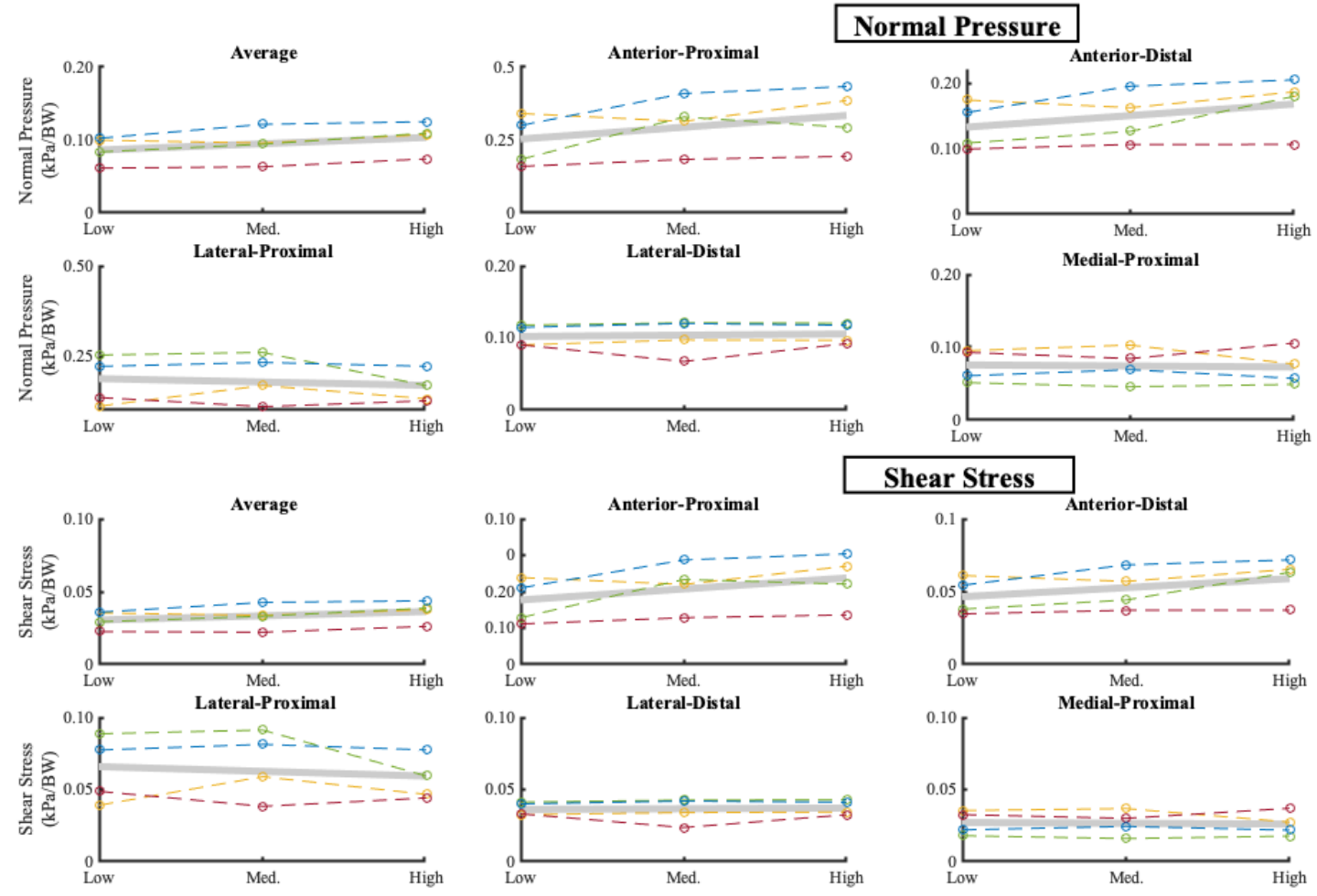

Shear Stress
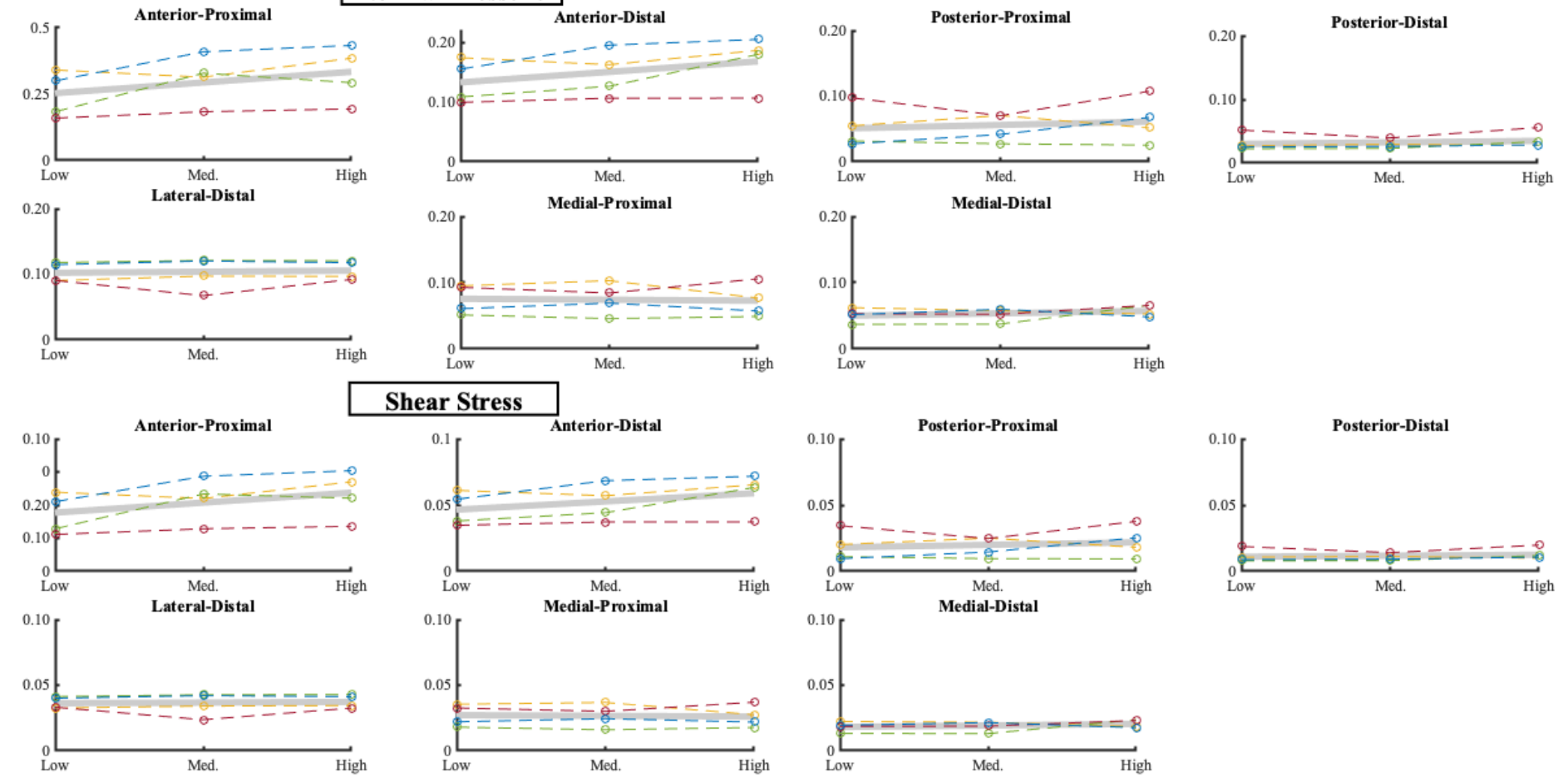
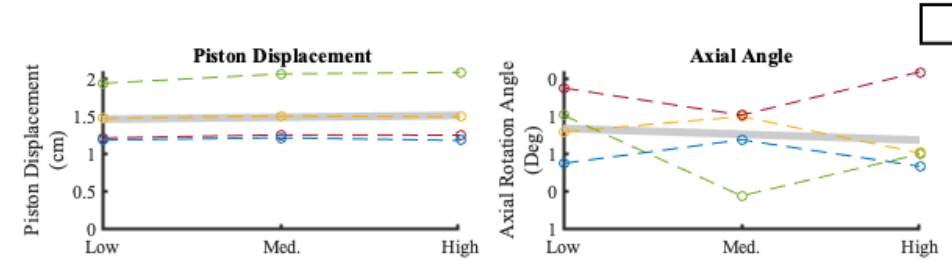

Figure 4: LME regression responses for normal forces (top), shear stresses (middle), and kinematics (bottom).

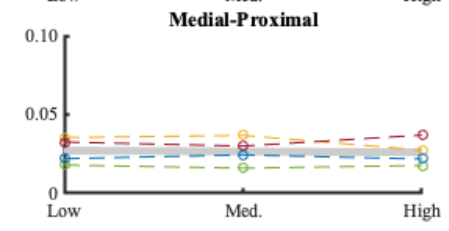

Kinematics
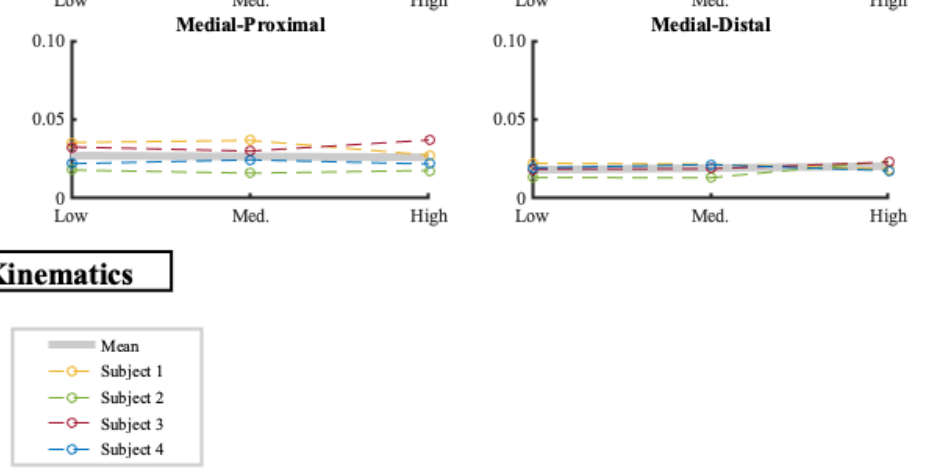

 
619 Table 3: MLE statistical parameters

\begin{tabular}{lllll}
\hline Outcome metric & Slope & Mean Intercept & Adjusted R & p-value \\
\hline \multicolumn{1}{l}{ Normal Pressure (kPa/BW) } & & & \\
\hline Peak Average & 0.009 & 0.078 & 0.742 & $0.003^{*}$ \\
Anterior Proximal & 0.040 & 0.213 & 0.524 & $0.027^{*}$ \\
Anterior Distal & 0.018 & 0.115 & 0.552 & $0.022^{*}$ \\
Posterior Proximal & 0.005 & 0.046 & 0.115 & 0.371 \\
Posterior Distal & 0.002 & 0.028 & 0.191 & 0.240 \\
Lateral Proximal & -0.009 & 0.196 & 0.092 & 0.428 \\
Lateral Distal & 0.002 & 0.100 & 0.052 & 0.554 \\
Medial Proximal & -0.001 & 0.077 & 0.025 & 0.686 \\
Medial Distal & 0.004 & 0.047 & 0.130 & 0.340 \\
\hline Shear Stress (kPa/BW) & & & \\
\hline Peak Average & 0.003 & 0.028 & 0.678 & $0.006^{*}$ \\
Anterior Proximal & 0.015 & 0.073 & 0.555 & $0.021^{*}$ \\
Anterior Distal & 0.006 & 0.040 & 0.552 & $0.022^{*}$ \\
Posterior Proximal & 0.002 & 0.016 & 0.116 & 0.371 \\
Posterior Distal & 0.001 & 0.010 & 0.151 & 0.302 \\
Lateral Proximal & -0.003 & 0.069 & 0.097 & 0.416 \\
Lateral Distal & $<0.001$ & 0.036 & 0.031 & 0.651 \\
Medial Proximal & -0.001 & 0.028 & 0.025 & 0.685 \\
Medial Distal & 0.001 & 0.017 & 0.106 & 0.393 \\
\hline Kinematics (Varying Units) & & & 0.557 \\
\hline Axial Angle (deg) & -0.078 & 0.426 & 0.052 & 0.103 \\
Pistoning (cm) & 0.025 & 1.446 & 0.334 & \\
\hline *Stastically signicant & $\alpha-0.05)$ & & & \\
\hline
\end{tabular}


Figures and Tables
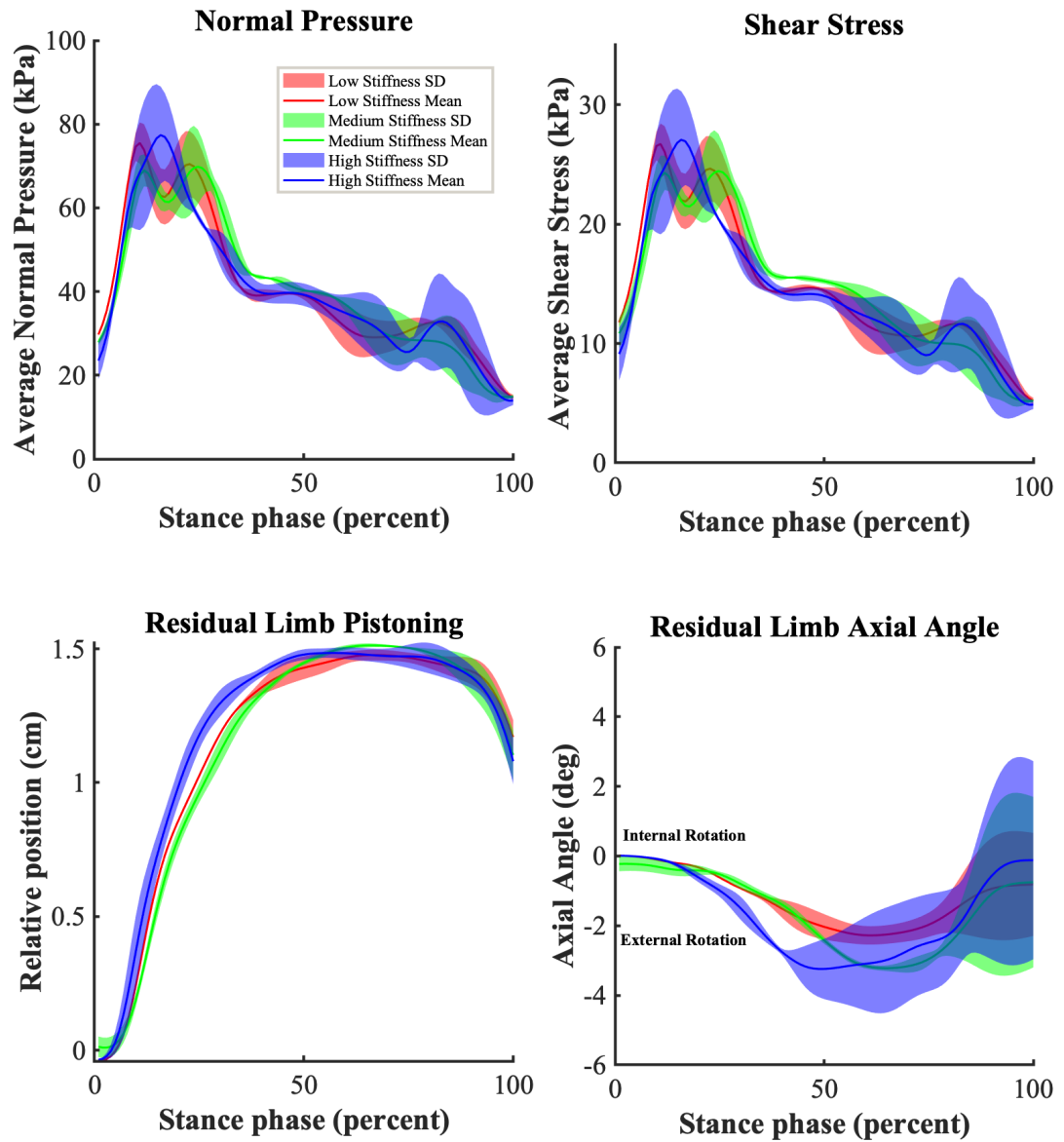

Figure 5: Mean data for normal pressure, shear stress, residual limb pistoning, and residual limb internal/external rotation across stance phase for the low, medium, and high stiffness conditions. Kinetic data are normalized to subject body weight. 
Figures and Tables
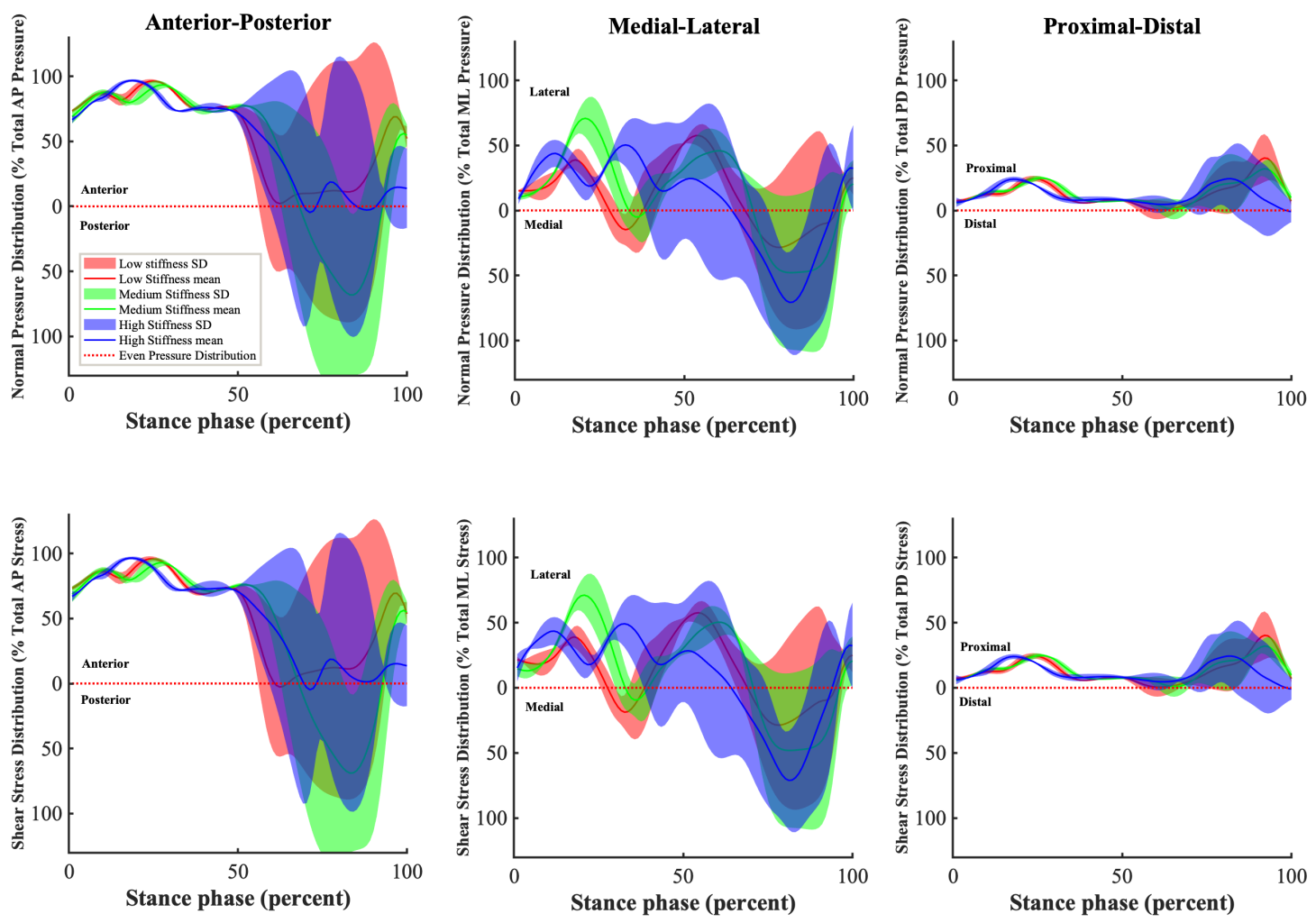

Figure 6: Subject 1 mean data for normal pressure (top) and shear stress (bottom) distributions in the anterior-posterior, medial-lateral, and proximal-distal directions. Pressure and shear 630 stress distributions are the percent contribution of opposing interface frames (AP, ML, and PD) to the sum of the pressure or shear stress of the opposing the interfaces 
Table 3: Peak average values for normal and shear stress (average of nine anatomical locations), and peak values for residual limb piston displacement with respect to the prosthetic socket. Data are mean \pm SD.

\begin{tabular}{llll}
\hline $\begin{array}{l}\text { Subject } \\
\text { (condition) }\end{array}$ & $\begin{array}{l}\text { Normal Pressure } \\
(\mathbf{k P a})\end{array}$ & $\begin{array}{l}\text { Shear Stress } \\
(\mathbf{k P a})\end{array}$ & $\begin{array}{l}\text { Piston } \\
\text { Displacement }(\mathbf{c m})\end{array}$ \\
\hline $\mathbf{1}$ (low) & $75.7 \pm 3.71$ & $26.8 \pm 1.26$ & $1.48 \pm 0.02$ \\
$\mathbf{1}$ (medium) & $72.7 \pm 6.42$ & $25.5 \pm 2.22$ & $1.51 \pm 0.01$ \\
$\mathbf{1}$ (high) & $81.54 \pm 4.11$ & $28.5 \pm 1.44$ & $1.50 \pm 0.03$ \\
$\mathbf{2}$ (low) & $91.2 \pm 10.93$ & $32.0 \pm 3.84$ & $1.95 \pm 0.10$ \\
$\mathbf{2}$ (medium) & $103 \pm 6.47$ & $36.1 \pm 2.30$ & $2.07 \pm 0.15$ \\
$\mathbf{2}$ (high) & $119.2 \pm 32.6$ & $42.1 \pm 11.7$ & $2.09 \pm 0.19$ \\
$\mathbf{3}$ (low) & $50.5 \pm 1.08$ & $18.6 \pm 0.47$ & $1.22 \pm 0.01$ \\
$\mathbf{3}$ (medium) & $52.1 \pm 0.61$ & $18.2 \pm 0.19$ & $1.26 \pm 0.02$ \\
$\mathbf{3}$ (high) & $61.0 \pm 13.9$ & $21.5 \pm 4.89$ & $1.26 \pm 0.02$ \\
$\mathbf{4}$ (low) & $64.1 \pm 1.41$ & $22.5 \pm 22.5$ & $1.19 \pm 0.00$ \\
$\mathbf{4}$ (medium) & $76.1 \pm 4.24$ & $36.6 \pm 1.50$ & $1.22 \pm 0.03$ \\
$\mathbf{4}$ (high) & $78.3 \pm 1.26$ & $27.4 \pm 0.42$ & $1.19 \pm 0.03$ \\
Group (low) & $70.4 \pm 4.28$ & $25.0 \pm 1.52$ & $1.46 \pm 0.03$ \\
Group & $78.1 \pm 4.44$ & $26.6 \pm 1.55$ & $1.52 \pm 0.05$ \\
(medium) & $85.0 \pm 13.0$ & $29.9 \pm 4.61$ & $1.51 \pm 0.07$ \\
Group (high) & & & \\
\hline & & & \\
\hline
\end{tabular}


Table 4: Average peak values for normal pressure $(\mathrm{kPa})$ by anatomical location, subject, and condition. A zero value for the distal contact interface implies that the distal tibia did not contact the base of the prosthetic socket (i.e., piston displacement $<1.5 \mathrm{~cm})$. Data are mean \pm SD.

\begin{tabular}{|c|c|c|c|c|c|c|c|c|c|}
\hline $\begin{array}{l}\text { Subject } \\
\text { (condition) }\end{array}$ & $\begin{array}{l}\text { Anterior } \\
\text { Proximal }\end{array}$ & $\begin{array}{l}\text { Anterior } \\
\text { Distal }\end{array}$ & $\begin{array}{l}\text { Posterior } \\
\text { Proximal }\end{array}$ & $\begin{array}{l}\text { Posterior } \\
\text { Distal }\end{array}$ & $\begin{array}{l}\text { Medial } \\
\text { Proximal }\end{array}$ & $\begin{array}{l}\text { Medial } \\
\text { Distal }\end{array}$ & $\begin{array}{l}\text { Lateral } \\
\text { Proximal }\end{array}$ & $\begin{array}{l}\text { Lateral } \\
\text { Distal }\end{array}$ & Distal \\
\hline 1 (low) & $259 \pm 30.6$ & $132 \pm 6.52$ & $41.5 \pm 24.9$ & $20.6 \pm 4.86$ & $72.9 \pm 29.9$ & $47.4 \pm 1.61$ & $84.1 \pm 2.25$ & $68.6 \pm 3.28$ & $0.08 \pm 0.06$ \\
\hline 1 (medium) & $238 \pm 41.9$ & $123 \pm 12.0$ & $53.4 \pm 19.4$ & $22.2 \pm 4.23$ & $78.1 \pm 44.4$ & $43.8 \pm 7.41$ & $128 \pm 17.6$ & $73.7 \pm 4.35$ & $4.91 \pm 5.34$ \\
\hline 1 (high) & $292 \pm 14.2$ & $141 \pm 6.99$ & $39.2 \pm 15.2$ & $20.9 \pm 2.74$ & $58.3 \pm 15.9$ & $40.0 \pm 2.19$ & $99.7 \pm 10.4$ & $73.3 \pm 4.95$ & $4.91 \pm 5.34$ \\
\hline 2 (low) & $199 \pm 19.2$ & $118 \pm 13.9$ & $34.5 \pm 6.84$ & $24.5 \pm 0.95$ & $56.0 \pm 11.3$ & $40.5 \pm 2.72$ & $275 \pm 32.4$ & $128 \pm 11.9$ & $89.5 \pm 33.8$ \\
\hline 2 (medium) & $358 \pm 44.3$ & $138 \pm 12.3$ & $29.9 \pm 17.9$ & $25.0 \pm 1.03$ & $49.9 \pm 11.2$ & $41.0 \pm 3.13$ & $283 \pm 52.4$ & $132 \pm 23.3$ & $145 \pm 39.7$ \\
\hline 2 (high) & $320 \pm 21.4$ & $196 \pm 49.4$ & $27.5 \pm 3.37$ & $36.6 \pm 11.7$ & $53.4 \pm 15.7$ & $71.7 \pm 26.4$ & $182 \pm 31.8$ & $131 \pm 38.4$ & $233 \pm 104$ \\
\hline 3 (low) & $131 \pm 14.5$ & $81.8 \pm 4.62$ & $80.1 \pm 5.52$ & $42.5 \pm 3.11$ & $76.5 \pm 4.08$ & $43.7 \pm 3.01$ & $111 \pm 16.6$ & $74.5 \pm 7.04$ & $0.00 \pm 0.00$ \\
\hline 3 (medium) & $151 \pm 7.19$ & $87.5 \pm 2.46$ & $57.7 \pm 8.35$ & $32.4 \pm 3.90$ & $69.4 \pm 12.5$ & $43.1 \pm 5.46$ & $90.1 \pm 13.1$ & $55.4 \pm 3.07$ & $0.00 \pm 0.00$ \\
\hline 3 (high) & $160 \pm 3.20$ & $87.7 \pm 3.86$ & $88.2 \pm 51.4$ & $45.7 \pm 26.0$ & $87.0 \pm 13.3$ & $54.1 \pm 7.38$ & $103 \pm 23.6$ & $75.7 \pm 25.1$ & $0.00 \pm 0.00$ \\
\hline 4 (low) & $188 \pm 12.5$ & $37.2 \pm 5.50$ & $17.1 \pm 0.72$ & $15.7 \pm 0.72$ & $38.1 \pm 2.53$ & $32.4 \pm 2.02$ & $138 \pm 6.97$ & $71.8 \pm 2.53$ & $0.00 \pm 0.00$ \\
\hline 4 (medium) & $256 \pm 25.0$ & $122 \pm 9.51$ & $26.1 \pm 6.90$ & $16.4 \pm 0.57$ & $43.3 \pm 3.56$ & $37.5 \pm 0.39$ & $145 \pm 5.49$ & $75.2 \pm 1.53$ & $0.00 \pm 0.00$ \\
\hline 4 (high) & $271 \pm 0.24$ & $128 \pm 0.92$ & $42.3 \pm 2.83$ & $17.8 \pm 0.78$ & $35.9 \pm 4.11$ & $30.3 \pm 2.20$ & $139 \pm 7.78$ & $73.6 \pm 3.19$ & $0.00 \pm 0.00$ \\
\hline Group (low) & $194 \pm 19.2$ & $107 \pm 7.65$ & $43.3 \pm 9.50$ & $25.8 \pm 2.41$ & $60.7 \pm 12.0$ & $41.0 \pm 2.34$ & $152 \pm 14.5$ & $85.8 \pm 6.19$ & $22.4 \pm 8.46$ \\
\hline Group (medium) & $251 \pm 29.6$ & $118 \pm 9.05$ & $41.8 \pm 13.1$ & $24.0 \pm 2.44$ & $60.2 \pm 17.9$ & $41.3 \pm 4.10$ & $162 \pm 22.2$ & $84.0 \pm 8.05$ & $36.9 \pm 10.0$ \\
\hline Group (high) & $261 \pm 9.77$ & $138 \pm 15.3$ & $49.3 \pm 18.2$ & $30.3 \pm 10.3$ & $58.6 \pm 12.3$ & $49.0 \pm 9.55$ & $131 \pm 18.4$ & $88.5 \pm 17.9$ & $59.5 \pm 27.4$ \\
\hline
\end{tabular}


Table 5: Average peak values for shear stress $(\mathrm{kPa})$ by anatomical location, subject, and condition. A zero value for the distal contact interface implies that the distal tibia did not contact the base of the prosthetic socket (i.e., piston displacement $<1.5$ $\mathrm{cm})$. Data are mean \pm SD.

\begin{tabular}{|c|c|c|c|c|c|c|c|c|c|}
\hline Subject (condition) & $\begin{array}{l}\text { Anterior } \\
\text { Proximal }\end{array}$ & $\begin{array}{l}\text { Anterior } \\
\text { Distal }\end{array}$ & $\begin{array}{l}\text { Posterior } \\
\text { Proximal }\end{array}$ & $\begin{array}{l}\text { Posterior } \\
\text { Distal }\end{array}$ & $\begin{array}{l}\text { Medial } \\
\text { Proximal }\end{array}$ & $\begin{array}{l}\text { Medial } \\
\text { Distal }\end{array}$ & $\begin{array}{l}\text { Lateral } \\
\text { Proximal }\end{array}$ & $\begin{array}{l}\text { Lateral } \\
\text { Distal }\end{array}$ & Distal \\
\hline 1 (low) & $90.5 \pm 10.7$ & $46.3 \pm 2.33$ & $15.4 \pm 8.13$ & $8.08 \pm 2.10$ & $26.9 \pm 9.58$ & $16.7 \pm 0.76$ & $29.7 \pm 1.01$ & $24.7 \pm 1.14$ & $0.03 \pm 0.02$ \\
\hline 1 (medium) & $83.1 \pm 14.7$ & $43.3 \pm 4.17$ & $19.0 \pm 6.27$ & $8.61 \pm 1.78$ & $27.9 \pm 15.1$ & $16.5 \pm 3.47$ & $44.7 \pm 6.25$ & $25.8 \pm 1.61$ & $0.98 \pm 0.23$ \\
\hline 1 (high) & $102 \pm 4.98$ & $49.5 \pm 2.44$ & $13.7 \pm 5.35$ & $7.51 \pm 0.96$ & $20.8 \pm 5.05$ & $14.1 \pm 0.82$ & $35.4 \pm 3.97$ & $26.1 \pm 1.74$ & $1.90 \pm 1.99$ \\
\hline 2 (low) & $69.9 \pm 6.71$ & $41.3 \pm 4.89$ & $12.1 \pm 2.34$ & $8.62 \pm 0.35$ & $19.6 \pm 3.95$ & $14.4 \pm 1.23$ & $96.5 \pm 11.4$ & $45.0 \pm 4.32$ & $35.8 \pm 12.9$ \\
\hline 2 (medium) & $127 \pm 18.1$ & $48.2 \pm 4.30$ & $10.5 \pm 6.25$ & $8.89 \pm 0.23$ & $17.5 \pm 3.92$ & $14.3 \pm 1.10$ & $99.3 \pm 18.5$ & $46.5 \pm 8.35$ & $51.7 \pm 13.1$ \\
\hline 2 (high) & $120 \pm 10.2$ & $68.9 \pm 17.5$ & $10.2 \pm 1.90$ & $12.9 \pm 4.18$ & $19.4 \pm 5.94$ & $25.7 \pm 9.65$ & $64.7 \pm 11.5$ & $46.7 \pm 13.9$ & $81.9 \pm 36.4$ \\
\hline 3 (low) & $45.8 \pm 5.10$ & $28.6 \pm 1.63$ & $28.3 \pm 1.97$ & $15.4 \pm 1.10$ & $26.8 \pm 1.43$ & $15.3 \pm 1.06$ & $40.1 \pm 5.32$ & $27.3 \pm 2.38$ & $0.00 \pm 0.00$ \\
\hline 3 (medium) & $52.8 \pm 2.51$ & $30.6 \pm 0.87$ & $20.4 \pm 2.91$ & $11.6 \pm 1.42$ & $24.7 \pm 4.76$ & $15.5 \pm 2.17$ & $31.5 \pm 4.58$ & $19.5 \pm 1.01$ & $0.00 \pm 0.00$ \\
\hline 3 (high) & $55.9 \pm 1.13$ & $30.7 \pm 1.34$ & $31.0 \pm 18.0$ & $16.3 \pm 8.99$ & $30.4 \pm 4.66$ & $19.0 \pm 2.69$ & $36.3 \pm 8.41$ & $26.8 \pm 8.95$ & $0.00 \pm 0.00$ \\
\hline 4 (low) & $65.7 \pm 4.37$ & $34.0 \pm 1.93$ & $6.01 \pm 0.27$ & $5.54 \pm 0.31$ & $13.8 \pm 0.69$ & $12.2 \pm 1.21$ & $48.3 \pm 2.43$ & $25.1 \pm 0.85$ & $0.00 \pm 0.00$ \\
\hline 4 (medium) & $89.8 \pm 8.74$ & $42.8 \pm 3.33$ & $9.11 \pm 2.42$ & $5.73 \pm 0.19$ & $15.3 \pm 1.37$ & $13.5 \pm 0.38$ & $50.9 \pm 1.93$ & $16.3 \pm 0.54$ & $0.00 \pm 0.00$ \\
\hline 4 (high) & $95.0 \pm 0.08$ & $44.9 \pm 0.32$ & $15.9 \pm 0.08$ & $6.71 \pm 0.73$ & $13.7 \pm 2.53$ & $11.0 \pm 0.37$ & $48.5 \pm 2.71$ & $25.8 \pm 1.16$ & $0.00 \pm 0.00$ \\
\hline Group (low) & $68.0 \pm 6.72$ & $37.6 \pm 2.70$ & $15.4 \pm 3.18$ & $9.41 \pm 0.97$ & $21.8 \pm 3.91$ & $14.7 \pm 1.06$ & $53.7 \pm 5.03$ & $30.5 \pm 2.17$ & $8.95 \pm 3.23$ \\
\hline Group (medium) & $88.2 \pm 11.0$ & $41.2 \pm 3.17$ & $14.7 \pm 4.46$ & $8.70 \pm 0.91$ & $21.4 \pm 6.28$ & $14.9 \pm 1.78$ & $56.6 \pm 7.82$ & $29.5 \pm 2.88$ & $13.2 \pm 3.33$ \\
\hline Group (high) & $93.3 \pm 4.09$ & $48.5 \pm 5.40$ & $17.7 \pm 6.33$ & $10.9 \pm 3.72$ & $21.1 \pm 4.54$ & $17.5 \pm 3.38$ & $46.2 \pm 6.59$ & $31.3 \pm 6.43$ & $21.0 \pm 9.58$ \\
\hline
\end{tabular}


644 Equations

645

646 eq. 1: $F_{n}=\left\{\begin{array}{cc}(k \times \delta)+(b \times \dot{\delta}) & \delta>0, \dot{\delta}>0 \\ k \times \delta & \delta>0, \dot{\delta}<0 \\ 0 & \delta<0\end{array}\right.$

647

$648 \boldsymbol{F}_{\boldsymbol{n}}$ : normal force

$649 \boldsymbol{k}$ : contact stiffness

$650 \boldsymbol{\delta}$ : penetration depth

651 b: contact damping coefficient

652

653

654 eq. 2: $F_{f}=\left\{\begin{array}{cc}F_{n} \times \mu_{\text {static }} & v_{\text {poc }}<v_{\text {threshold }} \\ F_{n} \times \mu_{\text {kinetic }} & v_{\text {poc }}>v_{\text {threshold }}\end{array}\right.$

655

$656 \boldsymbol{F}_{f}$ : frictional force

$657 \mu$ : coefficient of friction

$658 v_{\text {poc }}$ : velocity at point of contact

$659 v_{\text {threshold }}$ : velocity threshold

660

661

662

eq. 3: $k_{n}=\frac{E A}{l}$

663

$664 \boldsymbol{k}_{n}$ : Stiffness in the normal plane

$665 E$ : Young's modulus of the tissue

666 A: Area of the contact point

667 I: Width of the residual limb 